Review paper

\title{
Radiation dose optimization in diagnostic and interventional radiology: Current issues and future perspectives
}

\author{
Virginia Tsapaki \\ Dosimetry and Medical Radiation Physics Section, International Atomic Energy Agency, Austria
}

\section{A B S T R A C T}

The medical radiological imaging technological evolution and wide availability has resulted in the exponential increase in utilization. Evidence for a risk of cancer arising from radiation doses of lower than $100 \mathrm{mSv}$ is still limited. There is a need for patient dose optimization. A literature review was conducted to identify the reasons why optimization is important and the steps to be followed for a successful optimization process in digital radiology, Computed Tomography, interventional radiology and mammography.

Based on recent literature, 5 steps for a dose optimization process are proposed. These steps are: 1) establishment of a quality assurance programme; a mistake, misuse or malfunction of an X-ray machine can potentially affect the health or life of thousands of people, 2) establishment of a dose optimization team consisting of a radiologist, medical physicist and radiation technologist, 3) determination of baseline dose levels and image quality as well as comparisons with benchmarks to decide which exam protocols should be optimized, 4) modification of protocols by the medical physicist and 5) evaluation of the optimization process and its effect on patient dose and image quality.

The optimization process should include joint efforts focused on 1) equipment performance 2) exam protocol customization and 3) staff behaviour. Manufacturers should provide detailed descriptions of exam protocols and training on the use of dose reduction features. The diagnostic radiology medical physicist should emerge and take a proactive lead in the everyday clinical routine in order to promote the value of optimization process.

\section{Introduction}

Medical imaging plays a pivotal role in accurate disease diagnosis and improved treatment of the patient and has proven to be invaluable to the whole process. Its use is vital at all levels of health care, in preventive medicine, curative and palliative care. In the last 50 years, the medical community has experienced a technology evolution that has boosted the use of ionizing radiation from X-ray equipment shifting steadily from analogue to digital detectors and platforms, from single slice to multidetector-row computed tomography (CT), from fluoroscopy to complex sophisticated angiography systems and from simple intraoral dental machines to panoramic and cone beam CT technologies. The wide accessibility of modern X-ray medical imaging together with increased patient demand has resulted in their use outside the traditional radiology department by numerous other clinical specialists such as interventional cardiologists, orthopaedic surgeons, gastroenterologists, dentists, anaesthesiologists, urologists, etc. [1].

The availability of imaging services is highly variable between countries but major medical institutions in capital cities tend to offer the most modern imaging modalities, almost regardless of the per capita gross national income [2]. The increasing use of medical imaging has resulted in growing utilization trends. The UNSCEAR 2008 Report presents results of a 1997-2007 survey and confirms rising trends in all Health Care levels mainly due to increasing use of CT [3]. The recently published National Council on Radiation Protection and Measurements (NCRP) report 184 [4] also confirmed an increase use in radiological procedures in the United States of America (USA) to 2016, albeit less marked than the rapid and dramatic increase of the early 1980s to 2006 [5]. According to a recent study that analysed data of more than 135 million imaging exams in USA and Ontario, Canada over the period 2000-2016, the annual increase in CT, MRI, and ultrasound exams was highest in the earlier years (i.e. between 2000 and 2006), but continued to rise between 2012 and 2016 by $1-5 \%$ annually for most age groups $[6]$.

The use of ionizing radiation in medical imaging is however associated with a risk of cancer and should therefore be subject to standards of safety and for this reason, be optimized [7]. The International Atomic Energy Agency (IAEA) Radiation Protection and Safety in Medical Uses of Ionizing Radiation, Safety Standards Series No. SSG-46 states that in diagnostic and interventional medical exposure, optimization is ".. keeping the exposure of patients to the minimum necessary to achieve the required diagnostic or interventional objective" [8].

\footnotetext{
* Address: Dosimetry and Medical Radiation Physics Section, International Atomic Energy Agency, Wagrammer Str 5, 1220 Vienna, Austria.

E-mail address: v.tsapaki@iaea.prg.
} 
The objectives of this review are therefore to discuss the issue of radiation dose optimization in diagnostic and interventional radiology taking into consideration all the above, suggest steps to follow to achieve this goal and finally, to discuss challenges and future perspectives

\section{Optimization: Why is it so important?}

\subsection{Current evidence on low radiation dose risk}

Evidence of radiation induced cancer is limited for radiation doses lower than $100 \mathrm{mSv}$. The level of cancer risk from diagnostic radiology is still a subject of discussion with no firm consensus within the scientific community even for CT, which is considered one of the high dose diagnostic procedures [9-15]. The only direct epidemiological data come from about 30,000 A-bomb survivors who were on the peripheries of Hiroshima and Nagasaki, and who were exposed to a similar low dose range [11]. This low-dose subpopulation has been followed for more than 70 years and shows a small but statistically significant increased cancer risk. The two large historical cohort studies [12,13] that used clinical data both for exposure and outcome seem to provide the strongest data so far but are still questioned by the scientific community $[9,10]$. Two European research projects are receiving substantial funding from the European Commission (EC) to address the issue of low dose radiation risk: 1) The "Implications of Medical Low Dose Radiation Exposure" (MEDIRAD) European Project is a 4-year project aimed at enhancing the scientific bases and clinical practice of radiation protection in the medical field [16] and 2) the "Epidemiological study to quantify risks for paediatric computerized tomography and to optimise doses" (EPI-CT) is an ongoing European project set-up to investigate the relationship between the exposure to ionizing radiation from CT scans in childhood and adolescence, and late health effects [17]. Unfortunately, results are not yet available. The Multidisciplinary European Low Dose Initiative (MELODI) [18], a European Platform dedicated to low dose radiation risk research and the European Alliance for Medical Radiation Protection Research (EURAMED) [19] that represents associations involved in the application of ionising radiation in medicine are working complementary within Europe in this direction. Finally, the most comprehensive reports that provide estimates of risk related to low radiation dose are the BEIR VII 2006 report [20] and the UNSCEAR 2013 [21] report focused on children. These reports are commonly cited and recommend the use of the so-called "linear no-threshold model" (LNT) as the most reasonable relationship between low-dose exposure to ionizing radiation and the incidence of solid cancers induced by ionizing radiation. This implies that a single $\mathrm{X}$-ray photon is capable of inducing cancer. The implication is that the optimization process is vital for the protection of patients and especially for particular patient groups (paediatric, pregnant or patients that need to have repeat exams due to specific clinical conditions).

\section{Patient radiation dose levels}

With increasing utilization, variety, and complexity of X-ray procedures the next inevitable question is the level of radiation dose associated with them. This is important for the strategic planning of the optimization process. Most radiological examinations are in the range of sub $\mathrm{mSv}$ (chest X-ray) to approximately $10-15 \mathrm{mSv}$ (CT or interventional procedures) [22]. Technological improvements have offered the possibility to lower radiation dose from medical imaging. The international literature has focused mostly on CT due to higher doses compared to other X-ray techniques and to recent injuries reported [1,10-22]. Patients and the media are also discussing the issue of radiation dose in CT resulting in exaggerated articles, causing excessive stress to patients, or even convincing patients to decline a CT scan that could have a positive health impact [9]. The scientific community and the CT industry reacted to this by expanding and concentrating research on more accurate estimation of radiation dose and optimization tools. The special report of the Summit on Management of Radiation Dose in CT in 2011 identified various steps to reduce the effective dose from a routine single-phase abdomen and pelvis CT from $10 \mathrm{mSv}$ to less than $1 \mathrm{mSv}$, i.e. one-third of average annual dose from background radiation [22]. Despite the enormous benefit of optimizations tools, unfortunately there is "no easy fix at a national level or a corporate vendor level for the need to select and implement optimized protocols within the thousands of hospitals and imaging centers that offer CT imaging" [10]. This is also evident in fluoroscopy and interventional procedures where new sophisticated machines offer numerous examination protocols, that are preset by the manufacturer for easiness of use, but proper use is not guaranteed [23]. Even if one assumes that the protocol settings are optimum for the clinical task, knowledge of how they work in practice is needed for efficient use of tools offered by the manufacturer and for successful optimization. Unfortunately, there is no universal implementation.

\section{Optimization process}

\subsection{Step 1: Establishment of a quality assurance program}

The causes of excessively high patient doses are usually attributed either to poor equipment conditions, or to poor optimization of exam protocols [24]. The first step in a successful optimization process is to have an established quality assurance program. The World Health Organization (WHO) has defined the quality assurance program as an organized effort that ensures that images produced during an X-ray imaging procedure are of a sufficiently high quality that they consistently provide adequate diagnostic information at the lowest possible cost and with the least possible exposure of the patient to radiation [25] The optimization process requires a balance between patient dose and image quality along with other clinical considerations, including the use of a contrast agent [24].

The IAEA and EC have published several reports on quality assurance and dosimetry in diagnostic radiology [24,26-30]. Quality control reports are produced by professional medical physics societies such as the American Association of Physicists in Medicine (AAPM) [31-33], the Institute of Physics and Engineering in Medicine (IPEM) of United Kingdom (UK) $[34,35]$ or the European Federation of Organisations for Medical Physics (EFOMP) [36]. Whatever protocol or guidance is followed, a quality assurance program must first be established to ensure effective patient dose optimization.

\subsection{Step 2: Define the optimization team}

An optimization team should be established consisting of radiologists, medical physicists and radiation technologists (radiographers). Each of these professionals has a unique role in the optimization process. The radiologist provides feedback on whether sufficient task-specific image quality is maintained, the medical physicist guides the optimization process by applying various methods described elsewhere $[37,38]$ or in many institutions actually optimizes the exam protocols keeping sufficient image quality at the appropriate radiation dose [39] and the radiation technologist ensures that the modified (optimized) exam protocol is feasible in the clinical workflow and executed correctly [39].

The team will decide which are the most important clinical tasks, exam types and for which X-ray modalities optimization is needed from the radiation exposure point to view. As the number of modalities (digital radiography, CT, mammography, fluoroscopy, dental, etc), exam types (depending usually on anatomical region and clinical task) and frequency of exams differ substantially across medical institutions, this should be decided at an institutional level. Once the exam types are decided, then clinically appropriate image quality requirements must be established in collaboration with clinicians. 


\subsection{Step 3: Define baseline}

A baseline of patient dose levels for the exam types decided by optimization team must then be established preferably performing appropriate dose measurements. This must be done simultaneously with image quality assessment based on the criteria set in Step 2. Usually the image quality criteria include noise/spatial resolution measurements, which should be evaluated by the radiologists. Patient dose measurements are performed by the clinically qualified medical physicist using standard dose metrics [29] and standard procedures [40]. There is a wide variety of dose metrics depending on the X-ray modality. Once assessed, dose values must be compared with local or national diagnostic references levels (DRLs) or other relevant benchmark values taking also into account image quality evaluation $[7,8,40]$. The optimization team will then conclude which of the examinations require optimization. According to a recent study, reviewing all protocols at a single institution could require the full-time effort of a dedicated individual for a full year [41].

DRLs are proven to be an important tool for optimization. According to the IAEA BSS, they represent the level of dose above which it is judged to be inappropriate to plan to allow exposures to occur, and below which the optimization of protection and safety is implemented. The DRL value chosen will depend on the circumstances for the exposures under consideration $[7,8,40]$. ICRP among other organizations underlines the importance of DRLs and gives clear guidance on how to define DRLs for radiological procedures [40]. Often however, there is misinterpretation in their use; there is an assumption that if patient doses are below DRL this means adequate optimization or there were even problems with DRL implementation [42,43]. All these should be taken into consideration when establishing DRLs. Another approach was recently introduced named as the acceptable quality dose (AQD). This quantity is based on the clinically acceptable image quality as the primary goal rather than only the patient radiation dose (each facility can determine average dose values for various examinations that have images of clinically acceptable quality by well-informed imaging specialists that are classified in weight groups of $10 \mathrm{~kg}$ body weight) for adults [43].

\subsection{Step 4: Modification of exam protocols}

The modification of exam protocols for radiation dose optimization purposes is a complex task that needs to be performed by the medical physicist [39] in collaboration with the other members of the team once steps $1-3$ are finalized $[37,38]$. Medical technology today offers numerous tools to achieve this goal but this should be attempted only if in-depth knowledge of machine performance is acquired and how each technical parameter, post processing algorithm or other feature can affect image quality and radiation dose, is considered. Thus, the medical physicist will need to study all technical documentation to understand each machine's features and optimization tools [39]. The manufacturer's application specialists can provide advanced knowledge, best practice, and tips and tricks for the specific modality in question [39].

\subsection{Digital radiography}

Traditional radiographic imaging dose optimization methods per examination are increasing $\mathrm{kV}$, decreasing $\mathrm{mAs}$, reducing exposure time, appropriate positioning and using collimation to image only the body region required for the organ in focus. With the introduction of digital radiology, the potential of dose reduction is enhanced by the dynamic range of digital detectors. Digital images can be evaluated using software provided by the manufacturer, free software such as ImageJ [44]. The appearance of the image can be improved with functions such as panning, zooming, inverting the grey scale, measuring distance and angle, and windowing. Image processing is one of the key features of digital radiography, greatly influencing the way the image appears [45]. However, until adjustment to the new digital environment is accomplished, there is potential for increasing radiation dose mainly due to the perceived lack of visual control [2]. Currently image processing techniques and noise reduction algorithms add to the traditional optimization strategies for optimal radiographic imaging [46,47]. As already mentioned earlier monitoring of patient doses (in terms of exposure indices or relevant dose quantityies such as the Kerma-Area Product KAP) assists in optimization of all X-ray examinations and also radiographic procedures.

\section{6. $C T$}

Currently many articles in the literature suggest various strategies for CT protocol optimization, e.g. tube modulation, $\mathrm{kV}$ modulation in smaller size and younger patients, z-axis overscan, scan length limitation, use of iterative reconstruction algorithms, use of dual energy (particularly important for contrast enhanced exams in patients with renal function problems), use of machine learning and artificial intelligence algorithms [22,48-54]. Cardiac high-pitch CT angiography of neonates with congenital heart diseases can be performed safely and with dose reduction without additional test bolus or bolus tracking scans [55]. Demb et al 2017 provided a comprehensive optimization step approach by analyzing 158,274 diagnostic CT scans and concluding that by reviewing institutional doses and sharing dose-optimization best practices, lower radiation doses for chest and abdominal CT, and more consistent doses for head CT were reported [56].

\subsection{Fluoroscopy}

Many publications focus on radiation protection issues in fluoroscopy and specially in interventional procedures [1,57-66]. The common feature of all studies is that patient dose optimization in interventional procedures occurs by a combination of equipment protocol modification and operator behaviour, and not simply by changing the protocol characteristics. The active use of digital processing, customization of exam protocols, removal of antiscatter grid in specific clinical cases (such as spinal digital subtraction angiography), reduction of pulse rate and frame rate, default use of the fluoroscopy mode at low level, as well as continuous dose monitoring and patient follow-up, are suggested. The new technology features that currently accompany state-of-the art X-ray angiography machines provide potential for dose optimization but their use and their effect on radiation dose must be clearly understood $[62,63]$. A recent study by Tsalafoutas et al has shown that protocol setting has a large impact on image characteristics and radiation exposure, and that these protocols do not always perform as expected [23]. Thorough investigation of X-ray machines during commissioning and routine quality control by the medical physicist is vital.

\subsection{Mammography}

As mammographic imaging is performed to asymptomatic women for early detection of breast cancer, optimization of dose is of paramount importance. Furthermore, the new technologies introduced the last years such as breast tomosynthesis and contrast enhanced mammography, increase the use of mammographic imaging whereas methods to accurately estimate breast dose are lacking. Ultra-low-dose craniocaudal images in digital mammography have potential in the screening of a largely young and asymptomatic population and provide a baseline for further research in optimization of a low-dose approach [67].

\subsection{Step 5: Evaluation of optimization process: Effect in patient dose and image quality}

Given a period for adjustment to newly modified exam protocols, step 3 must be repeated in order to evaluate the effect of the optimization process. Recent literature cites numerous publications that are related to the optimization process and patient dose reductions 
[41,46-69]. For example, Smith-Bindman R et al applied customized optimization steps in 100 facilities for 864080 adult patients and $1,156,657$ CT scans, and reported that detailed feedback on CT radiation dose combined with actionable suggestions and quality improvement education significantly reduced doses, whereas the effects of audit feedback alone were modest [68]. The same authors in another study that included hospitals from around the world (over 2 million CT examinations of adult patients from 151 institutions in Switzerland, Netherlands, Germany, United Kingdom, United States, Israel, and Japan), concluded that the dose variation across countries was primarily attributable to institutional decisions regarding the technical parameters of CT protocols rather than patient, institution or machine characteristics [69].

\section{Discussion}

There is consensus among international organizations around the world such as IAEA, International Commission on Radiological Protection (ICRP), UNSCEAR, WHO, [57,70,71] or international campaigns such as Arabsafe [72], Eurosafe Imaging [73], Image Gently [74], Image Wisely [75] and Latinsafe [76], that patient radiation protection is a very important subject. The IAEA supports activities to educate and train professionals on all items related to patient dosimetry and radiation protection. Furthermore, expertise and technical support is provided through a specific International Action Plan for the Radiation Protection of Patients [57]. Many organizations, professional societies, countries, and individuals have joined these efforts, leading to the creation of the Bonn Call for Action [77]. The EC has supported efforts for research related to patient radiation dose optimization. EURAMED, already mentioned, is a platform for research activities harmonise clinical practice to advance the European radiation protection safety culture in medicine [19]. The ICRP provides extensive and very significant guidance on radiation protection in medical uses of ionizing radiation recent examples being, the report on Radiological protection in cardiology [78] and on Managing Patient Dose in Multi-Detector Computed Tomography [79]. A lot of efforts and resources (human and financial) have been provided over the last 30 years to develop a patient safety culture and low dose radiology apparatus though joint efforts at a global level $[16,17,74,80,81]$.

Patient dose optimization is not a straightforward or simple process. Dose reduction cannot be achieved by following simple steps or by altering technical parameters to reduce radiation exposure. The complexity of machines, the pace of technology evolution, the post processing possibilities and software algorithms, together with sophisticated exam protocols make the process quite demanding. Manufacturers have provided radiation protection tools and committed to dose reduction [22]. As an example, the association of the Heads of European Radiological Protection Competent Authorities (HERCA) and the European Association representing the medical imaging, electromedical and healthcare IT industry (COCIR) are working together to reduce patient radiation exposure in CT. They have focused on initiatives to improve dose reporting, to continue reducing the number of medical exposures, and provide specific training curricula [82]. Despite the availability of tools, resistance to change CT protocols, can be an important barrier to dose optimization [83].

When an X-ray machine is installed, training is provided by the manufacturer as agreed during the procurement process. In most cases, the training is focused on the basic operation principles of the systems and not on the use of the tools to obtain the best image quality with the lowest amount of radiation dose. If the equipment is to be used outside the radiology department, such as a surgical theatre, then the stress of environment can force personnel to start working with a system without time spent to "learn" all the doe reduction tools that the system allows. Occasionally, additional training is provided until users feel comfortable with the use of devices. Practical experience has shown however that even if installation engineers and/or application specialists are willing to assist, they are not responsible for optimization tools applied to everyday clinical practice $[23,84]$. As the optimization process relies on a complex combination of equipment performance, protocol modification and staff behaviour, the role as well as knowledge and skills of the clinically qualified medical physicist becomes more crucial in this process and requires active engagement in the routine daily clinical environment [85-87]. As stated in the recent European Federation of Organizations for Medical Physics (EFOMP) statement, the diagnostic radiology medical physicist clinical activities should be to carry out, participate in and supervise everyday activities related to patient safety and quality to ensure on-going effective and optimised use of medical devices, including patient specific optimization, prevention of unintended or accidental exposures and patient follow-up [86].

For the future, the use of new technology tools and their role in radiation dose optimization needs to be thoroughly investigated. Examples of recent such tools are a) the Radiation Dose Structured Report (RDSR), b) the patient dose management software (PDMS) or c) artificial intelligence (AI). As specifically mentioned in the paper of Sechopoulos et al. in 2015, the RDSR is a valuable tool during optimization of image acquisition protocols and can be used to directly inform national dose registries without relying on the information provided in image headers [88]. The authors suggest using the RDSR to get the information necessary to perform patient and foetal dose estimates [88]. Recent literature underlines the importance of PDMS on optimization $[40,84,89]$ and mentions that they will play a major role in ensuring best practice ensuring appropriate image quality at the required radiation dose [88]. Recently the use of artificial intelligence (AI) is also proposed as a tool for dose optimization [90]. In 2019, ESR published a white paper on what should the radiologist know about AI [91]. The white paper mentions specifically that AI can be used as an optimising tool to assist the technologist and radiologist in choosing a personalised patient's protocol, tracking the patient's dose parameters, providing an estimate of the radiation risks.

\section{Conclusion}

Medical imaging has clearly transformed the methods clinicians apply to address various clinical problems, treat patients and deal with life-threatening diseases. However, a mistake, misuse or malfunction of an X-ray machine can affect the health or the life of thousands of people and not just one X-ray systems must be closely monitored using established quality assurance programs and the use of machines should be tailored to each individual patient's needs with the appropriate quality and dose. The optimization process should include joint efforts of key professionals and activities focused on 1) equipment performance 2) exam protocol customization and 3) staff behaviour. The role of medical industry is to offer more radiation dose optimization tools and provide training not only on basic operation of their X-ray machines, but also on the application of preset exam protocols. Finally, the clinically qualified diagnostic radiology medical physicist should engage fully in the everyday medical imaging activities within the hospital and take the lead in the optimization process.

\section{References}

[1] International Commission on Radiological Protection (ICRP), 2010. Radiological Protection in Fluoroscopically Guided Procedures outside the Imaging Department. ICRP Publication 117, Ann. ICRP 40(6).

[2] International Atomic Energy Agency (IAEA). Worldwide Implementation of Digital Imaging in Radiology, Human Health Series No. 28, IAEA, Vienna (2015).

[3] United Nations Scientific Committee on the Effects of Atomic Radiation (UNSCEAR) 2008 Report to the General Assembly. "Sources and effects of ionizing radiation" Volume I; Annex A - Medical radiation exposures; United Nations, New York, 2013.

[4] National Council on Radiation Protection and Measurements (NCRP). Medical Radiation Exposure of Patients in the United States. Report No 184. Bethesda, Md: National Council on Radiation Protection and Measurements, 2019. 
[5] National Council on Radiation Protection and Measurements (NCRP). Ionizing radiation exposure of the population of the United States. Report No 160. Bethesda, Md: National Council on Radiation Protection and Measurements, 2009.

[6] Smith-Bindman R, Kwan ML, Marlow EC, et al. Trends in use of medical imaging in US health care systems and in Ontario, Canada, 2000-2016. JAMA 2019;322(9): 843-56. https://doi.org/10.1001/jama.2019.11456.

[7] European Commission, Food and Agriculture Organization of the United Nations, International Atomic Energy Agency, International Labour Organization, OECD Nuclear Energy Agency, Pan American Health Organization, United Nations Environment Programme, World Health Organization, Radiation Protection and Safety of Radiation Sources: International Basic Safety Standards: General Safety Requirements Part 3, No. GSR Part 3, IAEA, Vienna (2014).

[8] International Atomic Energy Agency (IAEA). Radiation Protection and Safety in Medical Uses of Ionizing Radiation, IAEA Safety Standards Series No. SSG-46, IAEA, Vienna (2018).

[9] Hendee WR, O'Connor MK. Radiation risks of medical imaging: separating fact from fantasy. Radiology 2012;264(2):312-21. https://doi.org/10.1148/ radiol.12112678.

[10] Thrall JH. Radiation exposure in CT scanning and risk: where are we? Radiology 2012 Aug;264(2):325-8. https://doi.org/10.1148/radiol.12121137.

[11] Brenner DJ, Hall EJ. Computed tomography: an increasing source of radiation exposure. N Engl J Med 2007;357(22):2277-84

[12] Pearce MS, Salotti JA, Little MP, et al. Radiation exposure from CT scans in childhood and subsequent risk of leukaemia and brain tumours: a retrospective cohort study. Lancet 2012;380(9840):499-505.

[13] Mathews John D, Forsythe Anna V, Zoe B, et al. Cancer risk in 680000 people exposed to computed tomography scans in childhood or adolescence: data linkage study of 11 million Australians. BMJ 2013;346:f2360.

[14] Zondervan R, Hahn P, Sadow C, Liu B, Lee S. Radiation from CT scanning young adults: what are the real risks? AJR Am J Roentgenol 2012;198(Suppl):56.

[15] Chen JX, Kachniarz B, Gilani S, Shin JJ. Risk of malignancy associated with head and neck CT in children: a systematic review. Otolaryngol Head Neck Surg 2014; 151(4):554-606.

[16] MEDIRAD project: Implications of Medical Low Dose Radiation Exposure. URL: http://www.medirad-project.eu, last assessed 5th September 2020.

[17] EPI-CT: International paediatric CT scan study. URL: http://epi-ct.iarc.fr/ , last assessed 17th June 2020

[18] Multidisciplinary European Low Dose Initiative (MELODI): http://www.melodionline.eu/index.html last assessed 5th September 2020.

[19] European Alliance for Medical Radiation Protection Research (EURAMED): https://www.euramed.eu/ last assessed 5th September 2020.

[20] National Research Council. Health Risks from Exposure to Low Levels of Ionizing Radiation: BEIR VII Phase 2. Washington, DC: The National Academies Press; 2006 https://doi.org/10.17226/11340.

[21] United Nations Scientific Committee on the Effects of Atomic Radiation (UNSCEAR) 2013 Report to the General Assembly. Volume II: Scientific Annex B: Effects of radiation exposure of children. United Nations, New York, 2013.

[22] McCollough CH, Chen GH, Kalender W, et al. Achieving routine submillisievert CT scanning: report from the Summit on Management of Radiation Dose in CT. Radiology 2012;264(2):567-80.

[23] Tsalafoutas IA, Tsapaki V and Triantopoulou S. Evaluation of image quality and patient exposure using phantoms: is there any clinical relevance? Personal communication.

[24] International Atomic Energy Agency (IAEA). Quality Assurance Programme for Computed Tomography: Diagnostic and Therapy Applications, Human Health Series No. 19, IAEA, Vienna, 2012.

[25] World Health Organization (WHO). Quality Assurance in Diagnostic Radiology. WHO, Geneva, 1982.

[26] International Atomic Energy Agency (IAEA). Quality Assurance Programme for Screen Film Mammography, Human Health Series No. 2, IAEA, Vienna, 2009.

[27] International Atomic Energy Agency (IAEA). Quality Assurance Programme for Digital Mammography, Human Health Series No. 17, IAEA, Vienna, 2011.

[28] International Atomic Energy Agency (IAEA). Status of Computed Tomography Dosimetry for Wide Cone Beam Scanners, Human Health Reports No. 5, IAEA, Vienna, 2011.

[29] International Atomic Energy Agency (IAEA). Dosimetry in Diagnostic Radiology: An International Code of Practice, Technical Reports Series No. 457, IAEA, Vienna, 2007.

[30] European Commission (EC). Criteria for Acceptability of Medical Radiological Equipment used in Diagnostic Radiology, Nuclear Medicine and Radiotherapy, Radiation Protection No. 162, Luxemburg, 2012.

[31] American Association of Physicists in Medicine (AAPM). Quality control in diagnostic radiology. Report of Task Group \#12 Diagnostic X-ray Imaging Committee. AAPM Report No. 74, AAPM, Madison, 2002.

[32] American Association of Physicists in Medicine (AAPM). Acceptance testing and quality control of Photostimulable Phosphor Imaging Systems. Report No. 93, AAPM, Madison, 2006a.

[33] American Association of Physicists in Medicine (AAPM). Assessment of Display Performance for Medical Imaging Systems, AAPM, Madison, 2005.

[34] Institute of Physicists and Engineers in Medicine (IPEM). The Commissioning and Routine Testing of Mammographic X-Ray Systems. IPEM Report No. 89, IPEM, York, 2005.

[35] Institute of Physicists and Engineers in Medicine (IPEM). Recommended Standards for the Routine Performance Testing of Diagnostic X-Ray Imaging Systems. Report 91, IPEM, York, 2005.
[36] European Federation of Organizations for Medical Physics (EFOMP. Quality control in digital mammography. EFOMP Mammo Working Group, EFOMP, 2015.

[37] International Atomic Energy Agency (IAEA). Roles and Responsibilities, and Education and Training Requirements for Clinically Qualified Medical Physicists, Human Health Series No. 25, IAEA, Vienna, 2013.

[38] Gingold EL. The medical physicist's role in radiation optimization. J Am Coll Radiol 2017;14(10):1335-6. https://doi.org/10.1016/j.jacr.2017.05.018.

[39] Sensakovic WF, Warden 4th DR, Bancroft LW. The link between radiation optimization and quality. J Am Coll Radiol 2017;14(6):850-1. https://doi.org/ 10.1016/j.jacr.2017.03.020.

[40] International Commission on Radiological Protection (ICRP), 2017. Diagnostic reference levels in medical imaging. ICRP Publication 135. Ann. ICRP 46(1).

[41] Duong P-A, Little BP. Dose tracking and dose auditing in a comprehensive computed tomography dose-reduction program. Semin Ultrasound CT MR 2014;35 (4):322-30.

[42] Rehani M. Dose surveys and DRLs: critical look and way forward. Radiat Prot Dosim 2015;165(1-4):67-9.

[43] Rehani M Limitations of diagnostic reference level (DRL) and introduction of acceptable quality dose (AQD). Br J Radiol 2015; 88: 1045.

[44] Schindelin J. Rueden CT, Hiner MC et al. The ImageJ ecosystem: An open platform for biomedical image analysis. Molecular Reproduction and Development, PMID 26153368, 2015.

[45] Körner M, Weber CH, Wirth S, et al. Advances in digital radiography: physical principles and system overview. Radiographics 2007;27(3):675-86.

[46] Lee W, Lee S, Chong S, et al. Radiation dose reduction and improvement of image quality in digital chest radiography by new spatial noise reduction algorithm. PLoS ONE 2020;15(2):e0228609.

[47] Takagi S, Yaegashi T, Ishikawa M. Dose reduction and image quality improvement of chest radiography by using bone-suppression technique and low tube voltage: a phantom study. Eur Radiol 2020;30(1):571-80.

[48] Thakur Y, McLaughlin PD, Mayo JR. Strategies for radiation dose optimization. Curr Radiol Rep 2013;V1:1-10.

[49] Tsapaki V, Rehani M, Saini S. Radiation safety in abdominal computed tomography. Semin Ultrasound CT MR 2010;31(1):29-38.

[50] Tsapaki V, Rehani M. Dose management in CT facility. Biomed Imaging Interv J 2007;3(2):e43.

[51] Greffier J, Frandon J, Pereira F, et al. Optimization of radiation dose for CT detection of lytic and sclerotic bone lesions: a phantom study. Eur Radiol 2020;30 (2):1075-8.

[52] Eberhard M, Alkadhi H. Machine learning and deep neural networks: applications in patient and scan preparation, contrast medium, and radiation dose optimization. J Thorac Imaging 2020;35(Suppl 1):S17-20.

[53] Kambadakone A. Artificial intelligence and CT image reconstruction: potential of a new era in radiation dose reduction. J Am Col Radiol 2020;V17(5):649-51.

[54] Szczykutowicz T. The CT Handbook: Optimizing Protocols for Today's FeatureRich Scanners, 2020. ISBN: 9780944838532.

[55] Schindler P, Kehl HG, Wildgruber M, et al. Cardiac CT in the preoperative diagnostics of neonates with congenital heart disease: radiation dose optimization by omitting test bolus or bolus tracking. Acad Radiol 2020;7(5):e102-8.

[56] Demb J, Chu P, Nelson T, et al. Optimizing radiation doses for computed tomography across institutions: dose auditing and best practices. JAMA Intern Med 2017;177(6):810-7.

[57] Tsapaki V, Balter S, Cousins C, et al. The International Atomic Energy Agency action plan on radiation protection of patients and staff in interventional procedures: achieving change in practice. Phys Med 2018;52:56-64.

[58] Panick C, Wunderle K, Sands M, et al. Patient radiation dose reduction considerations in a contemporary interventional radiology suite. Cardiovasc Intervent Radiol 2018;41(12):1925-34.

[59] Morris PP, Geer CP, Singh J, et al. Radiation dose reduction during neuroendovascular procedures. J Neurointerv Surg 2018;10(5):481-6.

[60] Orrù E, Mekabaty AE, Millan DS, et al. Removal of antiscatter grids for spinal digital subtraction angiography: dose reduction without loss of diagnostic value. Radiology 2020;295(2):390-6.

[61] Liu B, Hirsch JA, Li X, et al. Radiation dose monitoring for fluoroscopically guided interventional procedures: effect on patient radiation exposure. Radiology 2019; 290(3):744-9.

[62] Manu S, Suntharos P, Boyle GJ, et al. Radiation reduction in the pediatric catheterization laboratory using a novel imaging system. J Invasive Cardiol 2018; 30(1):28-33.

[63] Chambers CE, Awuor S. Reducing radiation dose: Equipment, procedure, and operator Perfecting the Trifecta. Catheter Cardiovasc Interv 2018;92(7):1237-8.

[64] Werner GS, Glaser P, Coenen A, et al. Reduction of radiation exposure during complex interventions for chronic total coronary occlusions: implementing low dose radiation protocols without affecting procedural success rates. Catheter Cardiovasc Interv 2017;89(6):1005-12.

[65] Gutiérrez-Barrios A, Camacho-Galán H, Medina-Camacho F, et al. Effective Reduction of Radiation Exposure during Cardiac Catheterization. Tex Heart Inst J 2019; 46(3): 167-171. Published 2019 Jun 1. doi:10.14503/THIJ-17-6548.

[66] Jones AK, Balter S, Rauch P, et al. Medical imaging using ionizing radiation: optimization of dose and image quality in fluoroscopy. Med Phys 2014;41:014301.

[67] Bluekens AM, Veldkamp WJ, Schuur KH, et al. The potential use of ultra-low radiation dose images in digital mammography-a clinical proof-of-concept study in craniocaudal views. Br J Radiol 2015;88(1047):20140626.

[68] Smith-Bindman R, Chu P, Wang Y, et al. Comparison of the effectiveness of singlecomponent and multicomponent interventions for reducing radiation doses in 
patients undergoing computed tomography: a randomized clinical trial [published online ahead of print, 2020 Mar 30]. JAMA Intern Med 2020;180(5):1-11.

[69] Smith-Bindman R, Wang Y, Chu P, et al. International variation in radiation dose for computed tomography examinations: prospective cohort study. BMJ 2019; 364: k4931. Published 2019 Jan 2. doi:10.1136/bmj.k4931.

[70] International Atomic Energy Agency (IAEA), Radiation Protection of Patients (RPOP). Available at: https://www.iaea.org/resources/rpop, last assessed 5th September 2020.

[71] Joint IAEA and WHO Position Statement on the Bonn Call-for-Action. https:// www.who.int/ionizing_radiation/about/med_exposure/en/index3.html, last assessed 5th September 2020.

[72] Arabsafe initiative. Available at: https://www.arabsafe.org/, last assessed 5th September 2020.

[73] The European Society of Radiology Eurosafe Imaging initiative. Available at: http://www.eurosafeimaging.org/, last assessed 5th September 2020.

[74] The Image Gently Alliance. Image Gently. Available at: https://www.imagegently. org/, last assessed 5th September 2020.

[75] The American College of Radiology and the Radiological Society of North America Joint Task Force on Adult Radiation Protection. Available at: https://www. imagewisely.org/, last assessed 5th September 2020.

[76] Latinsafe initiative. Available at: http://latinsafe.org/espanol/, last assessed 5th September 2020.

[77] International Atomic Energy Agency (IAEA), Bonn Call for Action Platform. Available at: https://www.iaea.org/resources/rpop/resources/bonn-call-foraction-platform, last assessed 5th September 2020.

[78] Cousins C, Miller DL, Bernardi G, et al. ICRP publication 120: radiological protection in cardiology. Ann ICRP 2013;42(1):1-125.

[79] ICRP. Managing patient dose in multi-detector computed tomography (MDCT). ICRP Publication 102. Ann ICRP 2007;37(1).

[80] Rehani MM, Melick ER, Alvi RM, et al. Patients undergoing recurrent CT exams: assessment of patients with non-malignant diseases, reasons for imaging and imaging appropriateness. Eur Radiol 2020;30(4):1839-46. https://doi.org/ 10.1007/s00330-019-06551-8.
[81] Rehani MM, Yang K, Melick ER, et al. Patients undergoing recurrent CT scans: assessing the magnitude. Eur Radiol 2020;30(4):1828-36. https://doi.org/ 10.1007/s00330-019-06523-y.

[82] CT Manufacturer's Voluntary Commitment Regarding CT Dose To HERCA Working Group "Medical Application"/Sub-Working Group "CT Manufacturers' involvement" Available at: https://www.herca.org/documents/COCIR\%20CT\% 20MANUFACTURER\%20Commitment $\% 20$ Version $\% 202 \% 20 \% 2013 \% 20$ May $\%$ 202011.pdf, last assessed 5th September 2020.

[83] Whitebird RR, Solberg LI, Bergdall AR, et al. Barriers to CT dose optimization: the challenge of organizational change. Acad Radiol 2020;S1076-6332(20):30102-11.

[84] EUCLID-European Study on Clinical Diagnostic Reference Levels for X-ray Medical Imaging. Available at: http://www.eurosafeimaging.org/euclid. Last assessed 5th September 2020 and personal communication.

[85] Kortesniemi M, Tsapaki V, Trianni A, et al. The European Federation of Organisations for Medical Physics (EFOMP) White Paper: big data and deep learning in medical imaging and in relation to medical physics profession. Phys Med 2018;56:90-3.

[86] Caruana CJ, Tsapaki V, Damilakis J, et al. EFOMP policy statement 16: the role and competences of medical physicists and medical physics experts under 2013/59/ EURATOM. Phys Med 2018;48:162-8.

[87] Delis H, Christaki K, Healy B, et al. Moving beyond quality control in diagnostic radiology and the role of the clinically qualified medical physicist. Phys Med 2017; 41:104-8.

[88] Sechopoulos I, Trianni A, Peck D. The DICOM radiation dose structured report: what it is and what it is not. J Am Coll Radiol 2015;V12(7):712-3.

[89] Parakh A, Kortesniemi M, Schindera ST. CT Radiation dose management: a comprehensive optimization process for improving patient safety. Radiology 2016; 280(3):663-73.

[90] Meineke A, Rubbert C, Sawicki LM, et al. Potential of a machine-learning model for dose optimization in CT quality assurance. Eur Radiol 2019;29:3705-13.

[91] Neri E, de Souza N, Brady A, et al. What the radiologist should know about artificial intelligence - an ESR white paper. Insights Imaging 2019;10:44. 